\title{
A randomized control trial comparing train of four ratio $>0.9$ to clinical assessment of return of neuromuscular function before endotracheal extubation on critical respiratory events in adult patients undergoing elective surgery at a tertiary hospital in Nairobi
}

\author{
Isaac Adembesa ${ }^{1}$, Vitalis Mung'ayi ${ }^{1}$, Zulfiqarali Premij ${ }^{2}$, Dorothy Kamya ${ }^{1}$
}

1. Department of Anaesthesia, Aga Khan University, East Africa.

2. Department of Pathology, Aga Khan University, East Africa.

\section{Author details:}

1. Isaac Adembesa- Phone: + 25420366 2158; Email: isaac.adembesa@aku.edu; 2. Zulfiqarali Premji- Phone: + 254203662158 ; Email: zulfiqarali.premji@aku.edu; 3. Dorothy Kamya- Phone: + 25420366 2158; Email: dorothy.kamya@aku.edu

\begin{abstract}
Background: There is increasing evidence that the incidence of postoperative residual paresis after using neuromuscular blockers ranges from 24 to $50 \%$ in post anaesthesia care unit (PACU) and is associated with postoperative complications such as critical respiratory events as evidenced by hypoxia, hypoventilation and upper airway obstruction. Quantitative neuromuscular monitoring (such as the assessment of Train of four (TOF) ratio) and reversal of neuromuscular blockers has been shown to reduce postoperative residual paresis. There are very few outcome studies on effect of residual paresis in PACU. There is a paucity of published randomized controlled trials investigating whether using a TOF ratio $\geq 0.9$ before endotracheal extubation compared to clinical assessment of return of neuromuscular function reduces the incidence of critical respiratory events in PACU.

Objective: To determine whether using TOF ratio $\geq 0.9$ compared to clinical assessment of return of neuromuscular function before endotracheal extubation reduces the incidence of critical respiratory events in PACU

Methods: Onehundred sixty eight adult patients in ASA physical status I and II requiring general anaesthesia for elective surgery with cisatracurium as the muscle relaxant were randomized into 2 groups of 84 each. Group 1 were patients who required a TOF ratio of $\geq 0.9$ before extubation. Group 2 patients were extubated based on clinical assessment of return of adequate neuromuscular function by the anaesthetist as is the standard of practice at the Aga Khan University hospital Nairobi. General anaesthesia was standardized in both groups. Both the investigators and patients were blinded during the study.

Once the patient was transferred to PACU, oxygen saturation (SP02), respiratory rate and any signs of upper airway obstruction as demonstrated by stridor, laryngospasms or requirement of any airway manipulation was recorded for the first 30 minutes. Duration of anaesthesia and surgery was also recorded. Patient demographics were recorded and analyzed.

Results: There was no statistical difference between the 2 groups in terms of patient demographics, duration of surgery and anaesthesia and duration since last muscle relaxant was given. In terms of hypoxia on arrival in PACU, the incidence of mild hypoxia (SPO2 90-93\%) was 11\% in clinical assessment groupversus $5 \%$ in TOF group P-value 0.149 while severe hypoxia (SPO2 <90\%) was $19 \%$ versus $10 \%$ P-value 0.078 . During the first 30 minutes in PACU, the incidence of mild hypoxia (SPO2 90-93\%) was statistically significant between the 2 groups (12\% in clinical assessment group versus $1 \%$ in TOF group, P-value 0.005$)$ while severe hypoxia (SPO2 <90\%) was 7\% versus 5\%, P-value 0.373. The incidence of upper airway obstruction was statistically significant between the two groups (45\% in clinical assessment group versus $14 \%$ in TOF group P-value $<0.0001$ for patients requiring airway maneuver, $21 \%$ versus $2 \%$ P-value $<0.0001$ for those who required tactile stimulation and $31 \%$ versus $12 \%$ were snoring, P-value 0.003 . Logistic regression analysis revealed TOF group was less likely associated with mild hypoxia (OR 0.09 95\% CI 0.01-0.71 P-value 0.023), tactile stimulation (OR 0.09 95\% CI 0.02-0.40 P-value 0.002), airway maneuver (OR 0.20 95\% CI 0.10-0.43 P-value <0.001) and snoring (OR 0.30 95\% CI 0.13-0.68 P-value 0.04).

Conclusion: Among this population, there is a lower incidence of critical respiratory events in PACU with the use of neuromuscular monitoring using TOF ratio $\geq 0.9$ to assess neuromuscular function before endotracheal extubation compared with the use of clinical assessment methods.
\end{abstract}

Keywords: Randomized control trial, neuromuscular function, elective surgery, Nairobi.

DOI: https://dx.doi.org/10.4314/ahs.v18i3.40

Cite as: Adembesa I, Mung'ayi V, Premji Z, Kamya D. A randomized control trial comparing train of four ratio $>0.9$ to clinical assessment of return of neuromuscular function before endotracheal extubation on critical respiratory events in adult patients undergoing elective surgery at a tertiary hospital in Nairobi. Afri Health Sci. 2018;18(3): 807-816. https://dx.doi.org/10.4314/ahs.v18i3.40

\section{Corresponding author:}

Vitalis Mung'ayi,

Department of Anaesthesia, Aga Khan University,

East Africa. Phone: +254 203662175

Email: vitalis.mung'ayi@aku.edu; mungayi@gmail.com

\section{Introduction}

Muscle relaxation is required for tracheal intubation and to facilitate surgical procedures as part of balanced anaesthesia $^{1}$. If it is not adequately reversed after surgery, residual paresis leads to muscle weakness, respiratory

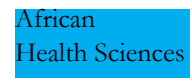

807
(C) 2018 Adembesa et al. Licensee African Health Sciences. This is an Open Access article distributed under the terms of the Creative commons Attribution License (https://creativecommons.org/licenses/BY/4.0), which permits unrestricted use, distribution, and reproduction in any medium, provided the original work is properly cited.
African Health Sciences Vol 18 Issue 3, September, 2018 
problems, prolonged stay in post anaesthesia care unit ( PACU), and delayed recovery ${ }^{2,3}$.

On recovery from muscle relaxation, the anaesthesiologist can assess for return of muscle power using a clinical criteria that includes the ability to lift the head for 5 seconds or hold a tongue depressor between the teeth. These clinical methods are unreliable and may be affected by other factors such as sedation or inability of the patient to follow instructions ${ }^{4}$.

When neuromuscular monitoring is used, qualitative methods such as tactile or visual assessment of the degree of neuromuscular block are inaccurate ${ }^{5}$. It is therefore clear that in addition to monitoring neuromuscular block clinically, quantitative methods such as acceleromyography, mechanomyography or electromyography should also be used ${ }^{4}$.

Residual muscle paresis is defined by a Train of four (TOF) ratio $<0.9$ with correlating signs and symptoms of muscle weakness ${ }^{6}$. Postoperative residual paresis can be reduced by monitoring neuromuscular blockade in the operating room ${ }^{7}$. A recent study by Eikermann et al showed that mild degrees of residual muscle paresis (TOF ratios of 0.7-0.9) can be associated with profound impairment of respiratory and pharyngeal muscle function6. This may be associated with respiratory events and aspiration in PACU. Therefore, complete recovery of muscle function should be present before tracheal extubation to avoid these risks.

PACU critical respiratory event has been defined as "any unanticipated hypoxemia (haemoglobin oxygen saturation $<90 \%$ ), hypoventilation (respiratory rate $<8$ breaths/minute) or upper airway obstruction (stridor or laryngospasm) requiring an active or specific intervention (ventilation, airway manipulation, insertion of an oral/ nasal airway, opioid or muscle relaxant reversal or tracheal intubation)"

Most studies done globally show that postoperative pulmonary complications come second to postoperative nausea and vomiting in terms of PACU complications?. A meta-analysis by Naguib et al demonstrated that the use of a neuromuscular monitor is not associated with a decreased incidence of postoperative residual paresis ${ }^{10}$. However, this meta-analysis relied heavily on cohort studies and the authors recommended more randomised control trials on this subject matter.

Neuromuscular monitoring is done irregularly in spite of availability of neuromuscular monitors in developed and developing countries. The reason for this practice is not due to financial capability but may be due to lack of knowledge among medical staff on availability of other methods of monitoring neuromuscular blockade. The other reason is often the lack of confirmed data and published research on the topic.

This study was designed determine whether using TOF ratio $\geq 0.9$ compared to clinical assessment of return of neuromuscular function before endotracheal extubation reduces the incidence of critical respiratory events in PACU. Our study question was: does neuromuscular monitoring using TOF ratio $\geq 0.9$ compared to clinical assessment of return of neuromuscular function before endotracheal extubation reduce critical respiratory events in PACU?

We hypothesized that there would be a decrease in the incidence of critical respiratory events in PACU when neuromuscular monitoring using TOF ratio $\geq 0.9$ is used before endotracheal extubation compared to clinical assessment of return of neuromuscular function. Our primary objective was to determine whether using TOF ratio $\geq 0.9$ compared to clinical assessment of return of neuromuscular function before endotracheal extubation reduces the incidence of critical respiratory events in PACU. Our secondary objectives were determine incidence and severity of hypoxia in PACU and to determine incidence of upper airway obstruction in PACU.

\section{Methods}

This study was performed following approval by the health research ethics committee of the Aga Khan University, East Africa. It was a double blinded prospective randomized control trial. Since Nairobi is a cosmopolitan city, the patients served by the hospital cut across most racial groups present within the country. Patients were recruited from the pre-anaesthesia clinics, which run on a daily basis, wards and day-care unit. The study population included all adults aged between 18 and 65 years undergoing elective surgery at Aga Khan University hospital Nairobi. The sample population included 168 ASA I and II adult patients undergoing elective surgery under general anaesthesia.

All adults, aged 18-65 year American society of anaesthesiology (ASA) physical status I and II undergoing elective surgery under general anaesthesia were included in this study. Reasons for exclusion were: 
1. ASA status III and above

2. Pregnant women

3. Patients with neuromuscular diseases

4. Patients on medications that interfere with neuromuscular junction function. For example, magnesium sulphate and aminoglycosides

5. Patients with renal, hepatic or respiratory diseases

6. Patient refusal to take part in the study

7. Patients with $\mathrm{BMI}>30$

8. Patients allergic to cisatracurium

9. Patients undergoing brain surgery

10. Children and the elderly
A sample size of 168 patients (84 per group) was sufficient to demonstrate $11 \%$ difference in the incidence of hypoxia and upper airway obstruction between the TOF ratio group and clinical assessment group at 95\% confidence level and a power of $80 \%$. We therefore hypothesized there would be a difference of at least $11 \%$ between the TOF ratio group and clinical assessment group. Patients were informed of the nature of the study and recruited if eligibility criteria were met. Eligible patients received written and oral explanations on the purpose and nature of the study. The patients who gave written informed consent were enrolled into the study and their files tagged with a special colour sticker for ease of identification. Participant flow diagram is shown in figure 1.

Figure 1: Flow diagram of patient distribution

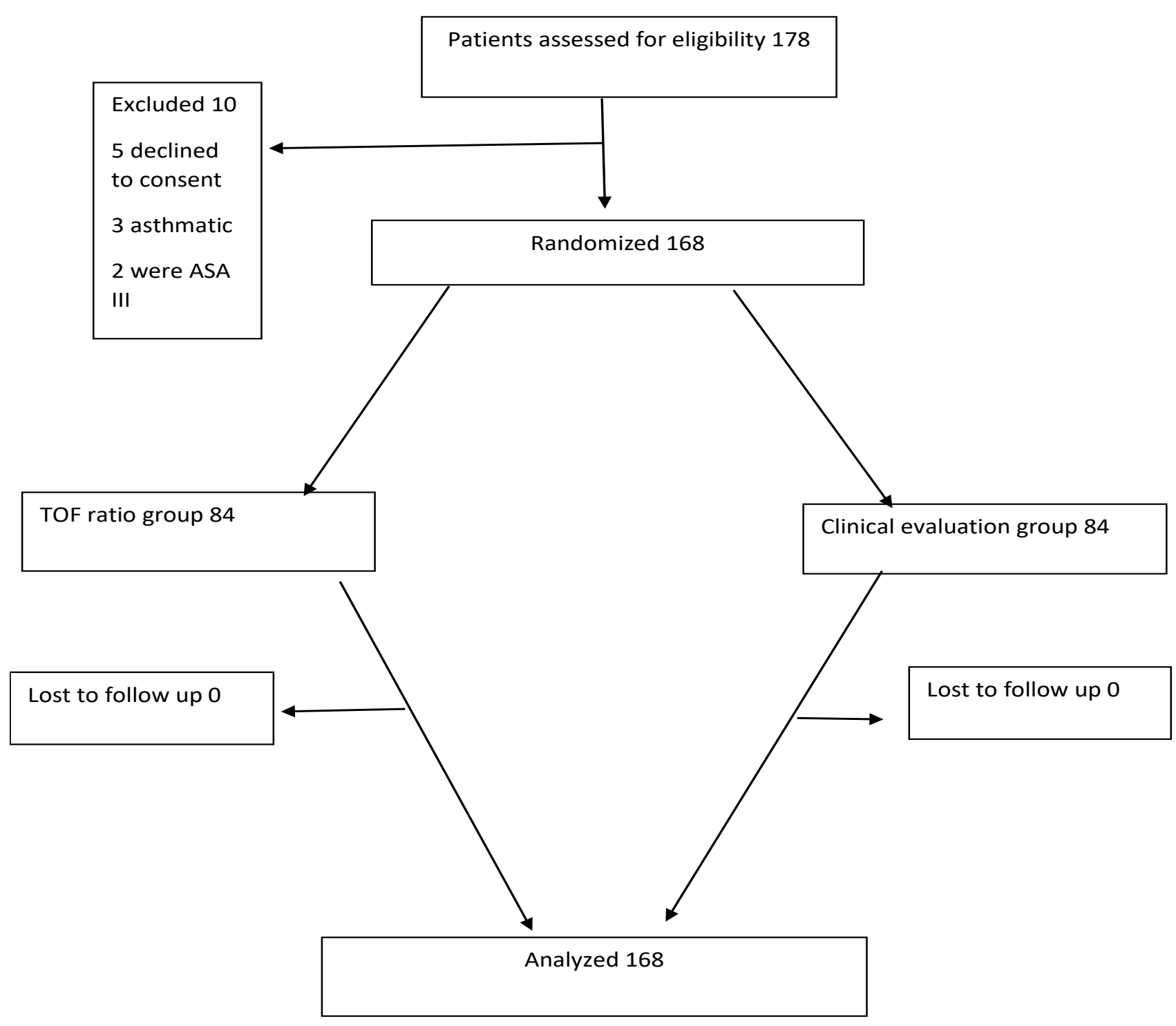


Simple randomization was generated by the statistician using a computer program. Each of the random numbers was sequentially assigned to either:

Group 1: TOF ratio

Group 2: Clinical assessment

These numbers were sealed in envelopes and delivered to the anaesthetist administering general anaesthesia. All physicians administering general anaesthesia were sensitized and trained on how to use the acceleromyographic nerve stimulator machine (TOF- WatchR SX, Organon). The principal investigator, research assistants and patients were not aware of the groups assigned.

General anaesthesia was standardized in the patients enrolled in the study. Upon entering theatre, the anaesthetist administering general anaesthesia was given a sealed envelope containing the group the patient had been randomised to. In the TOF ratio group, 2 surface electrodes were placed over the ulnar nerve at the wrist after cleansing and rubbing the skin with an alcohol solution. The acceleration transducer was then attached on the volar aspect of the distal phalanx of the thumb. Current selected was $50 \mathrm{~mA}$ for all the patients in this group (TOF watchR SX, Organon). This was done after the patient was under anaesthesia.

Intraoperative monitoring included non-invasive blood pressure, electrocardiography, pulse oximetry, capnography and core temperature monitoring via a nasopharyngeal temperature probe. Intravenous induction was done with $2 \mathrm{mcg} / \mathrm{kg}$ of fentanyl, $2-2.5 \mathrm{mg} / \mathrm{kg}$ propofol, and $0.2 \mathrm{mg} / \mathrm{kg}$ of cisatracurium. Orotracheal intubation was done after two to three minutes of bag ventilation and thereafter ventilation was controlled with a tidal volume of 6 to $8 \mathrm{mls} / \mathrm{kg}$, respiratory rate was varied to maintain end-tidal carbon dioxide between $30-35 \mathrm{mmHg}$.

Anaesthesia was maintained by isoflurane 1-2\% in oxygen and air to maintain oxygen saturations above $98 \%$. End tidal isoflurane was adjusted to keep blood pressure within $20 \%$ of baseline values. Any drop in blood pressure below this recommended level was corrected with 3-6mg boluses of ephedrine. Muscle relaxation was maintained with $0.05 \mathrm{mg} / \mathrm{kg}$ of cisatracurium boluses every 30 to 45 minutes as is the routine practice in our hospital. Last top-up of muscle relaxant was at least 30 minutes before end of anaesthesia.
Patients received standard analgesia of morphine $0.1 \mathrm{mg} /$ $\mathrm{kg}$, paracetamol $15 \mathrm{mg} / \mathrm{kg}$ and with a non-steroidal anti-inflammatory agent unless contra-indicated. Ondansetron $4 \mathrm{mg}$ with or without dexamethasone $8 \mathrm{mg}$ was given to all patients as prophylaxis against postoperative nausea and vomiting. Patients were kept warm by warm blankets, infusion of warm fluids and ambient theatre temperature of 240C. Core temperature was maintained above 350C. During reversal, isoflurane and air were switched off and patient was put on $100 \%$ oxygen. Oropharyngeal suctioning was done. Neostigmine 50micrograms/kilogram to a maximum of $5 \mathrm{mg}$ and atropine $20 \mathrm{microgram} /$ kilogram were given.

In the TOF ratio group, the patient required a TOF ratio $\geq 0.9$ before beingextubated and transferred to post anaesthesia care unit. In the clinical assessment group, the patient required head lift for at least 5 seconds, tidal volume $4-6 \mathrm{ml} / \mathrm{kg}$ with a regular respiratory rate of at least 12 breaths or coughing or pulling on the orotracheal tube before being extubated. Extubation was followed by gentle oral suctioning after which the patient received $100 \%$ supplemental oxygen by face mask with pulse oximetry monitoring until just before being transferred to post anaesthesia care unit. This is the routine practice in our hospital.

The patient was then transferred to PACU when the anaesthetist was comfortable with their clinical status. During transport, no oxygen was given. This is routine practice in our hospital.

Once the patient got to PACU, a pulse oximeter was connected by the investigator who was blinded to the study and baseline SPO2 recorded. After that, all the patients received oxygen $6 \mathrm{~L} /$ minute via the Hudson mask as is the practice in the hospital. Sustained SPO2<90\% for at least 5 seconds with a good pulse oximetry waveform was considered severe hypoxemia while SPO2 90-93\% mild hypoxemia. SPO2 was recorded continuously for 30 minutes. Any features of airway obstruction such as stridor, laryngospasms, airway manipulation by chin lift, jaw thrust or requirement of an oral airway or tactile stimulation or re-intubation, were recorded.

Data was collected by the principal investigator or research assistant in the PACU using the data collection form. The collected data was counterchecked for com- 
plete entry by the principal investigator and then entered into an MS-Excel data base. Data was analyzed using STATA version 11. Data was presented in tables. In terms of patients' demographic data, median values were used to represent age and body mass index and Wilcoxon signed rank sum test was used to compare the two groups. Sex, ASA status, smoking and operative procedures were presented as proportions and Pearson chi square test was used to compare the two groups.

Perioperative variables such as duration of surgery and anaesthesia, duration since last muscle relaxant was given were presented as means with standard deviations and two sample t-test used to compare the two groups. Outcome variables such as hypoxia, respiratory rate and indicators of upper airway obstruction such as tactile stimulation, airway maneuvers, snoring and re-intubation were presented as proportions and Pearson chi square test used to compare the two groups. Logistic regression analysis using both univariate and multivariate analysis where applicable was used to test for association between hypoxia and upper airway obstruction and study arm, operative procedure, duration of anaesthesia, duration since last muscle relaxant and BMI. These were presented as odds ratios with $95 \%$ confidence intervals. P-value of less than 0.05 was considered as statistically significant.

\section{Results}

One hundred sixty eight patients fulfilled the inclusion criteria and wererandomized into the study. No patient was excluded because of violation of study protocol. There was no statistical difference between the two groups in terms of patient demographic data. However, among operative procedures, there were more urologic procedures in the clinical assessment group $(21 \%$ versus $8 \%$, P-value $0.027)$ than TOF group as shown in table 1.)

Table 1 shows patient characteristics

\begin{tabular}{|c|c|c|c|}
\hline & $\begin{array}{l}\text { Clinical assessment } \\
\text { group }\end{array}$ & TOF group & P-values \\
\hline Number & $N=84$ & $\mathbf{N}=\mathbf{8 4}$ & \\
\hline Sex, M:F & $38(45 \%), 46(55 \%)$ & $36(43 \%), 48(57 \%)$ & 0.756 \\
\hline Age in yrs & $37.5(29-47)$ & $37(30-44)$ & 0.8192 \\
\hline BMI & $25.5(22.4-27.3)$ & $24.65(22.45-27.2)$ & 0.9380 \\
\hline \multicolumn{4}{|l|}{ ASA status } \\
\hline I & $27(32 \%)$ & $23(27 \%)$ & 0.500 \\
\hline II & $80(95 \%), 4(5 \%)$ & $80(95 \%), 4(5 \%)$ & 1.000 \\
\hline \multicolumn{4}{|l|}{ Smoking, No/Yes } \\
\hline Operative procedures & $2(2 \%)$ & $3(4 \%)$ & 0.650 \\
\hline ENT & $23(27 \%)$ & $23(27 \%)$ & 1.000 \\
\hline General & $21(25 \%)$ & $30(36 \%)$ & 0.131 \\
\hline Gynecologic & $1(1 \%)$ & $5(6 \%)$ & 0.096 \\
\hline Maxillofacial & $1(1 \%)$ & $0(0 \%)$ & 0.316 \\
\hline Ophthalmologic & $19(23 \%)$ & $15(18 \%)$ & 0.442 \\
\hline Orthopedics & $17(21 \%)$ & $7(8 \%)$ & 0.027 \\
\hline Urologic & $0(0 \%)$ & $1(1 \%)$ & 0.316 \\
\hline Plastic & & & \\
\hline
\end{tabular}

\footnotetext{
Median and $25^{\text {th }}-75^{\text {th }}$ percentiles are given for age and BMI. Otherwise, numbers of patients (and \% of patients) are given.

P-values for patients' data with median were calculated using Wilcoxon rank sum test while those with proportions were calculated using Pearson Chi-square test.

BMI-Body mass index, ASA- American Society of Anaesthesiology, ENT-Ear, Nose and Throat
} 
Intraoperative variables such as duration of anaesthesia $(130+71$ mins versus $130+70$ mins, $P$ value 0.6994$), d u-$ ration of surgery $(114 \pm 66$ mins versus $119 \pm 67$ mins, $\mathrm{P}$ value 0.6018) and duration since last muscle relaxant (53 \pm 16 mins versus $53 \pm 15, \mathrm{P}$ value 0.9806 ) were not statistically significant between the two groups as shown in table 2.

\section{Table 2 shows Perioperative variables}

\begin{tabular}{|c|c|c|c|c|}
\hline & $\begin{array}{l}\text { Clinical } \\
\text { assessment } \\
\text { group }\end{array}$ & $\begin{array}{l}\text { TOF } \\
\text { group }\end{array}$ & $\begin{array}{l}95 \% \text { Confidence } \\
\text { intervals }\end{array}$ & $\begin{array}{l}\mathrm{P}- \\
\text { values }\end{array}$ \\
\hline \multirow[t]{3}{*}{ Anaesthetic duration (mins) } & $130 \pm 71$ & $134 \pm 70$ & -25.7 to 17.3 & 0.6994 \\
\hline & & & & \\
\hline & $114 \pm 66$ & $119 \pm 67$ & -25.8 to 15.0 & 0.6018 \\
\hline Duration since last muscle relaxant (mins) & $53 \pm 16$ & $53 \pm 15$ & -4.9 to 4.8 & 0.9806 \\
\hline
\end{tabular}

Patients with SPO2 values $>94 \%$ in post anaesthesia care unit were more in the TOF group than clinical assessment group (85\% versus $70 \%$ on arrival, P-value 0.016 and $94 \%$ versus $81 \%$ within 30 minutes, P-value 0.010 ) as shown in table 3 and 4. Hypoxia was not statistically significant between the two groups on arrival in PACU. However, while in PACU, mild hypoxia (90-93\%) was more in the clinical assessment group than in the TOF group $(12 \%$ versus $1 \%$, P-value 0.005$)$ as shown in table 4.

Table 3 shows SPO2 values on arrival in post anaesthesia care unit

Clinical assessment TOF group
group

\begin{tabular}{llll}
\hline No. with SPO2 $>94 \%$ & $59(70 \%)$ & $72(85 \%)$ & 0.016 \\
No. with SPO2 $90-93 \%$ & $9(11 \%)$ & $4(5 \%)$ & 0.149 \\
No. with SPO2 $<90 \%$ & $16(19 \%)$ & $8(10 \%)$ & 0.078 \\
\hline
\end{tabular}

Table 4 shows SPO2 values for first 30 minutes in the post anaesthesia care unit

\begin{tabular}{llll}
\hline & $\begin{array}{l}\text { Clinical assessment } \\
\text { group }\end{array}$ & \multicolumn{2}{l}{ TOF group P-values } \\
\hline No. with SPO2 $>94 \%$ & $68(81 \%)$ & $79(94 \%)$ & 0.010 \\
No. with SPO2 $90-93 \%$ & $10(12 \%)$ & $1(1 \%)$ & 0.005 \\
No. with SPO2 $<90 \%$ & $6(7 \%)$ & $4(5 \%)$ & 0.373 \\
& & & \\
\hline
\end{tabular}


In terms of upper airway obstruction in the PACU, 45\% of patients in the clinical assessment group compared to $14 \%$ in the TOF group required an intervention such as jaw thrust/chin lift, oropharyngeal airway (P-value $<0.0001) .21 \%$ versus $2 \%$ required tactile stimulation to maintain their airway (P-value $<0.0001) .31 \%$ versus $12 \%$ were snoring in PACU (P-value 0.003). This was statistically significant as shown in table 5 . There was no difference in respiratory rate between the two groups. No patient required re-intubation in PACU.

\section{Table 5 shows airway variables in the post anaesthesia care unit}

\begin{tabular}{llll}
\hline & $\begin{array}{l}\text { Clinical } \\
\text { assessment } \\
\text { group }\end{array}$ & TOF group & P-value \\
\hline No. requiring airway maneuver, Yes/No & $38(45 \%), 46(55 \%)$ & $12(14 \%), 72(86 \%)$ & $<0.0001$ \\
Tactile stimulation, Yes/No & $18(21 \%), 66(79 \%)$ & $2(2 \%), 82(98 \%)$ & $<0.0001$ \\
Snoring, Yes/No & $26(31 \%), 58(69 \%)$ & $10(12 \%), 74(88 \%)$ & 0.003 \\
Respiratory rate & $16 \pm 2$ & $17 \pm 3$ & 0.0890 \\
Re-intubation, Yes/No & $0(0 \%), 84(100 \%)$ & $0(0 \%), 84(100 \%)$ & 0.36 \\
\hline
\end{tabular}

\section{Discussion}

The time between extubation and recovery of TOF ratios $\geq 0.9$ represents a particular vulnerable period for adverse respiratory events ${ }^{11}$. Several other factors may be associated with residual paresis in PACU. These include duration of anaesthesia and surgery, type of muscle relaxant used and use of reversal agent for NMBA at end of anaesthesia. In this study, anaesthetic technique was standardized and neostigmine was given as reversal agent. Cisatracurium was used in both groups and duration of anaesthesia and surgery was not statistically significant. On patient demographics, there was no statistically significant difference between the two groups. However, in terms of operative procedures, there were many urologic cases in the clinical assessment group compared to the TOF group (21\% versus $8 \%$ P-value 0.027$)$. However, this study was not powered to look at the effect of individual surgical procedure on critical respiratory events. Other operative procedures were similar between the two groups.

There were many patients with SPO2 $>94 \%$ in the TOF group compared to clinical assessment group on arrival in
PACU and during the first 30 minutes (85\% versus $70 \%$, P-value 0.016 and $94 \%$ versus $81 \%$, P-value 0.010$)$. On the other hand, the incidence of mild hypoxia (SPO2 9093\%) in PACU was $12 \%$ in the clinical assessment group compared to $1 \%$ in the TOF group (P-value 0.005). Logistic regression analysis, table 6 , showed a patient was less likely to develop mild hypoxia if in the TOF group compared to clinical assessment group (unadjusted OR 0.09 95\% CI 0.01-0.71 P-value 0.023), adjusted OR 0.11 95\% CI 0.14-0.93 P-value0.043). The incidence of severe hypoxia $(\mathrm{SPO} 2<90 \%)$ was $7 \%$ versus $5 \%$ P-value 0.373 . This could be due to active interventions that are undertaken by PACU staff hence minimizing cases of severe hypoxia. However, this is clinically significant given that Murphy et al demonstrated $0 \%$ incidence of severe hypoxia in PACU when using acceleromyography intraoperatively for monitoring neuromuscular function ${ }^{11}$. This could be due to higher patient to nurse ratio in our PACU whereby one nurse takes care of two patients or even at times three patients. This is in comparison to recommendations of one nurse to one patient. In the Murphy study, one patient was recovered by one nurse in PACU. 


\begin{tabular}{|c|c|c|c|c|}
\hline & $\begin{array}{lr}\begin{array}{l}\text { Unadjusted } \\
\text { ratio }\end{array} & \begin{array}{r}\text { odds } \\
\text { confidence } \\
\text { interval) }\end{array} \\
\end{array}$ & P-value & $\begin{array}{l}\text { Adjusted odds ratio } \\
\text { (95\% confidence } \\
\text { interval) }\end{array}$ & P-value \\
\hline \multirow{7}{*}{$\begin{array}{l}\text { Study arm } \\
\text { Operative procedure } \\
\text { Anesthetic duration } \\
\text { Duration since last muscle relaxant } \\
\text { BMI }\end{array}$} & $\begin{array}{l}\text { Mild hypoxia (90- } \\
\text { 93\%) }\end{array}$ & 0.023 & \multirow{7}{*}{$\begin{array}{l}0.11(0.14-0.93) \\
1.80(1.18-2.74)\end{array}$} & \multirow{7}{*}{$\begin{array}{l}0.043 \\
0.006\end{array}$} \\
\hline & $0.09(0.01-0.71)$ & 0.002 & & \\
\hline & $1.90(1.25-2.86)$ & 0.281 & & \\
\hline & $1.00(1.01-1.01)$ & 0.548 & & \\
\hline & $1.01(0.97-1.05)$ & & & \\
\hline & & 0.441 & & \\
\hline & $1.29(0.68-2.43)$ & & & \\
\hline
\end{tabular}

\section{Tactile stimulation}

$\begin{array}{lll}\text { Study arm } & 0.09(0.02-0.40) & 0.205 \\ \text { Operative procedure } & 1.16(0.92-1.47) & 0.305 \\ \text { Anesthetic duration } & 1.00(0.997-1.01) & 0.797 \\ \text { Duration since last muscle relaxant } & 1.00(0.97-1.03) & \\ \text { BMI } & & 0.098\end{array}$

$1.54(0.92-2.55)$

$\begin{array}{lll}\text { Study arm } & \text { Airway maneuver } & <0.001 \\ \text { Operative procedure } & 0.20(0.10-0.43) & 0.107 \\ \text { Anesthetic duration } & 1.15(0.97-1.36) & 0.403 \\ \text { Duration since last muscle relaxant } & 1.00(0.997-1.01) & 0.420 \\ \text { BMI } & 1.00(0.97-1.01) & \\ & 1.33(0.94-1.86) & 0.104 \\ & & \\ \text { Study arm } & \text { Snoring } & 0.004 \\ \text { Operative procedure } & 0.30(0.13-0.68) & 0.717 \\ \text { Anesthetic duration } & 1.03(0.86-1.25) & 0.466 \\ \text { Duration since last muscle relaxant } & 1.00(1.00-1.01) & 0.918 \\ \text { BMI } & 1.00(0.98-1.02) & \\ & & 0.119\end{array}$

-Smoking was not analyzed due to the very small numbers per group (4 each). Adjusted odds ratios were not calculated for tactile stimulation, airway manoeuvre and snoring because the other variables were not statistically significant.

Operative procedure was associated with almost twice risk of developing mild hypoxia in PACU (unadjusted OR 1.90 95\% CI 1.25-2.86 P-value 0.002); adjusted OR 1.80 95\% CI 1.18-2.74 P-value 0.006). This is in agreement with a study by Pedersen et al who demonstrated increased pulmonary complications after major orthopaedic surgery $^{12}$.

The incidence of upper airway obstruction requiring an airway maneuver was $45 \%$ in the clinical assessment group and $14 \%$ in the TOF group (P-value $<0.0001) .21 \%$ of patients in the clinical assessment group compared to $2 \%$ in the TOF group required tactile stimulation to maintain their airway (P-value $<0.0001)$. More patients in the clinical assessment group snored compared to those in TOF group (31\% versus 12\%, P-value 0.003). Logistic regression analysis revealed patients in the TOF group were less likely to require tactile stimulation (unadjusted OR $0.0995 \%$ CI 0.02-0.40 P-value 0.02), less likely to require an airway maneuver (unadjusted OR 0.20 95\% CI 0.10 $0.43 \mathrm{P}$-value <0.001) and less likely to snore (unadjusted 
OR 0.30 95\% CI 0.13-0.68 P-value 0.004) while in PACU. No multivariate analysis was done because the other possible confounders were not statistically significant after univariate analysis.

With all the possible confounders adjusted for, it is clear that the main determinant of hypoxia and upper airway obstruction in PACU is residual muscle paresis as demonstrated by less incidence of hypoxia and upper airway obstruction in the TOF group. Neuromuscular monitoring using TOF ratio appears to reduce incidence of critical respiratory events in PACU.

\section{Strengths of the study}

Perusal of the literature appears to indicate that this is one of the few prospective randomized controlled study that have compared the impact train of $>0.9$ to clinical assessment prior to endotracheal extubation on critically respiratory events.this study will add to the body of literature and knowledge on prevention of respiratory events in PACU after use of muscle relaxants.

\section{Limitations of the study}

This study was conducted at a single centre involving a relatively snmall number of patientshence impacting on the generalizability of our results. This study used TOF stimulation that was uncalibrated.This did have a risk of showing a TOF count instead of a TOF ratio .This reduces its reliability to guide decisions on pharmacological antagonism of non-depolarising block or to exclude postoperative residual curarization ${ }^{13}$. This study also lacked an objective measure of recovery from general anaesthesia such as a bispectral monitor. This was because at our hospital, objective measurement of depth of anaesthesia is not routinely done and this study aimed to mimick normal clinical practice which is a reflection of anaesthesia practice in Kenya and the whole of Eastern and Central Africa. Lastly, the operative procedures were varied and we recommend the study to be done in patients undergoing same surgical procedure.

\section{Conclusion}

On the basis of the results of this study and in this patient population, the use of TOF $\geq 0.9$ before tracheal extubation is associated with decreased critical respiratory events in PACU compared to clinical assessment and this should be the standard of care in our operating theatres. Trial registration: PACTR201501001021110.

\section{Conflict of interest}

We have no conflict of interest.

\section{References}

1. Massó E, Sabaté S, Hinojosa M, Vila P, Canet J, Langeron $\mathrm{O}$. Lightwand tracheal intubation with and without muscle relaxation. Anesthesiology. 2006 Feb; 104(2):249-54. 2. Murphy GS.Residual neuromuscular blockade: Incidence, assessment and relevance in the postoperative period. Minerva Anestesiol. 2006;72:97- PubMed ;109.

3. Sauer M, Stahn A, Soltesz S, Noeldge-Schomburg $G$, Mencke T. The influence of residual neuromuscular block on the incidence of critical respiratory events. A randomised, prospective, placebo-controlled trial. Eur. J. Anaesthesiol. 2011.;28(12):842-8. PubMed. http://dx.doi. org/10.1097/EJA.0b013e328345cd11

4. Mcgrath CD, Mb H, Frca C. Monitoring of neuromuscular block. Continuing education in anaesthesia, critical care and pain. 2006;6(1):7-12.

5. Viby-Mogensen et al.Tactile and Visual Evaluation of the Response to train of four nerve stimulation. Anesthesiology. 1985;63:440-443. PubMed .

6. Eikermann M, Blobner M, Groeben H, Rex C, Grote T, Neuhäuser M, et al. Postoperative upper airway obstruction after recovery of the train of four ratio of the adductor pollicis muscle from neuromuscular blockade. Anesth. Analg. 2006 Mar;102(3):937-42. http://dx.doi. org/10.1213/01.ane.0000195233.80166.14

7. Baillard C, Gehan G, Larmignat P, Samama CM, Cupa M. Residual curarization in the recovery room after vecuronium. BrJ Anaesth. 2000;84(3):394-5.

8. Rose DK, Cohen MM, Wigglesworth DF, DeBoer DP.. Critical respiratory events in PACU. Anesthesiology. 1994; 81:410-418.

9. Peskett MJ. Clinical indicators and other complications in the recovery room or postanaesthetic care unit. Anaesthesia. 1999 Dec; 54(12):1143-9. http://dx.doi. org/10.1046/j.1365-2044.1999.01077.x

10. Naguib M, Kopman AF, Ensor JE. Neuromuscular monitoring and postoperative residual curarisation: a meta-analysis. Br. J. Anaesth. 2007 Mar;98(3):302-16.http:// dx.doi.org/10.1093/bja/ael386 
11. Murphy GS, Szokol JW, Marymont JH, Franklin M, Avram MJ, Vender JS. Residual paralysis at the time of tracheal extubation. Anesth. Analg. 2005 Jun;100(6):1840-5. http://dx.doi.org/10.1213/01.ANE.0000151159.55655. CB

12. Pedersen T, Viby-Mogensen J, Ringsted C. Anaesthetic practice and postoperative pulmonary complications.
Acta Anaesthesiol. Scand. 1992 Nov;36(8):812-8. http:// dx.doi.org/10.1111/j.1399-6576.1992.tb03570.x

13. Martin-Flores M, Gleed RD, Basher KL, Scarlett JM, Campoy L, Kopman AF. TOF-Watch ${ }^{\circledR}$ monitor: failure to calculate the train-of-four ratio in the absence of baseline calibration in anaesthetized dogs. Br. J. Anaesth. 2012 Feb; 108(2):240-4.https://doi.org/10.1093/bja/aer378 